\title{
Controlled Release of Herbicides Using Nano-Formulation: A Review
}

\author{
Happiness Ugbede Itodo * \\ Department of Chemistry, Federal University of Agriculture, PMB 2373 Makurdi, Nigeria
}

\begin{abstract}
Receive Date: 21 February 2019, Revise Date: 9 April 2019, Accept Date: 14 May 2019
Abstract: Intensive use of the herbicides and other classes of chemicals products in agricultural practices has resulted in serious environmental impact, which increased the level of the herbicide residues in natural water, soil, and foodstuffs. Controlled release nano-formulation of herbicides in which the herbicides are incorporated in a nano-sized matrix might be an excellent alternative over the conventional methods of herbicides application that usually employed to control the pathogens, weeds, and several pests, thereby limiting the amount of the active ingredient available for unwanted processes. This review presents a better approach using nanotechnology for controlled release formulation of herbicides in agriculture.
\end{abstract}

Key words: Controlled release; Herbicide; Nano-formulation; Nanotechnology.

DOI: $10.33945 /$ SAMI/JCR.2019.2.4

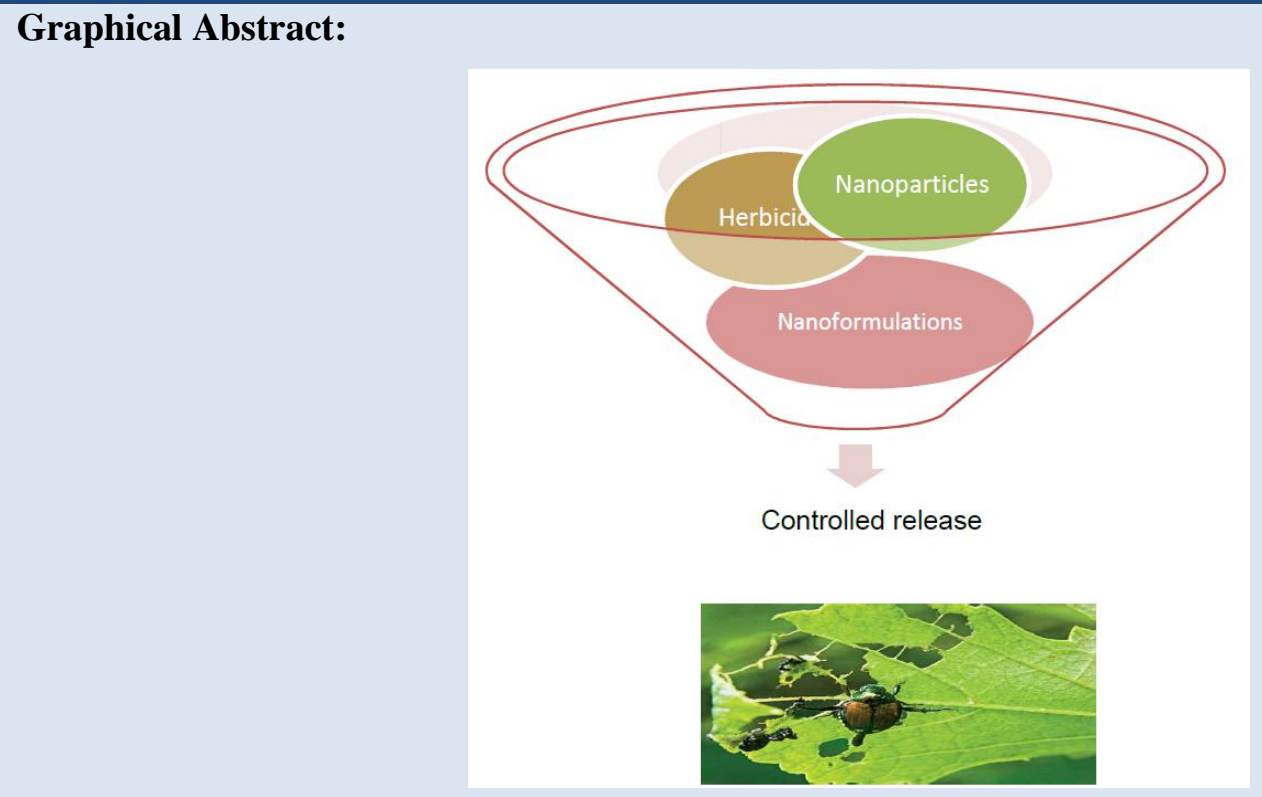

Biography:

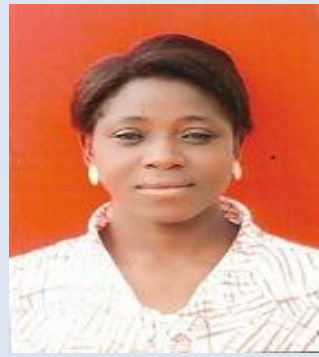

Happiness Itodo Ugbede: Itodo Ugbede Happiness, 36 years, married to Dr. Itodo Udoji Adams, hailed from Olamaboro Local Government Area of Kogi state. Itodo holds a BSc. in chemistry from Benue State University, Makurdi in 2010 after which she proceeded to the Federal University of Agriculture, Makurdi where she obtained her MSc. in Environmental Chemistry, 2016. She also had a post graduate diploma in Education (PGDE). Itodo research areas include Environmental chemistry, Analytical chemistry and Agrochemical technology. Itodo has authored and coauthored over twenty academic publications. 


\section{Introduction}

Agriculture which is also referred to farming which is the series of actions involve in the production of food, feed, fiber, and several other desired products by cultivating crops and raising livestock. This practice is the major backbone in most developing countries for providing food and other products for humans, either directly or indirectly [1]. Agriculture as a source of food is becoming increasingly important in a world of diminishing resources and an ever-increasing global population. The rapid growth in world's population with an estimated number of about 7.5 billion people at present and 9 billion by 2050 must all be fed, which means that global food productivity must increase to meet this urgent food demand [2]. Conventional methods employed in controlling pathogens, weeds, and several pests on agricultural farmlands and crops have a negative impact on both the environment and economy of farmers as $90 \%$ of the applied pesticides or herbicides are usually lost during application through volatilization, run-off and erosion thereby affecting the environment and higher application costs to the farmer [3]. To alleviate the adverse effects of pesticide residues on the environment, development of a more safe, economical, and reliable usage approach of pesticides is critically urgent [4]. A simple and effective method that can be used to reduce such effects is by incorporating herbicides in nanoparticles. Herbicide encapsulation aims at controlling the liberation of the formulation active ingredient, to enable their use for specific purpose at an intended concentration, reducing their disadvantages and maintain the highest possible effectiveness $[5,6]$. The major aim of the controlled release nanoformulations is to reduce application rate disadvantages on the environment and maintain highest possible effectiveness [7]. Advantages of the controlled release formulations have satisfactory effect at a constant active ingredient level for a longer period of time, smaller dosage as less active ingredient is needed to maintain effective biological activity thereby eliminating repeated applications and in the process reduce cost and longer application interval [8].

\section{Herbicides}

Herbicides are chemical substances that are utilized to specifically, partially or totally control or kill plants.
The mode of action for herbicides is the biochemical or physical mechanism, which includes absorption into plant and translocation to the site action and disrupting or altering one or more of their metabolic processes resulting in plant death [6]. Weeds always or predominantly grow in situations clearly altered by man, presenting undesirable effects at a specific time and place. According to reports [5], weeds are generally considered to hinder or alter the use of nutrients, water, light or useful space by the cultivated plants; have a series of biological advantages favoring invasion (easy seed dispersal) and persistence (high level of seed production, long period of feasibility, and stepwise germination) in cultivated zones; release substances through the roots and leaves which are toxic to crops, as they inhibit or hinder germination, growth or development of nearby plants (allelopathy); create a favorable habitat for other proliferating plagues (arthropods and ascari) by acting as a host; and hinder the normal harvest process and contaminate the production obtained [5].

Herbicides are required to control the biological and adaptive advantages of weeds [5]. They play important role in the production of agricultural yield by protecting the crops from pests. The large use of herbicides and other pesticides in agriculture causes serious environmental problems through leaching and run-off from the site of application [9]. The intensive use of herbicides and other classes of chemical products, in agricultural practice has resulted in serious impacts on the environment, causing an increase in the level of herbicide residues in natural water, soil, and foodstuffs $[10,11]$. The design of herbicides in nanoscale were intended for better plant protection from the damaging influences of weeds or plant diseases in comparison with the application of its bulk active ingredients [12]. The use of lower concentrations and fewer applications of herbicides is one of the prime objectives of the sustainable agriculture as it decreases the toxicity herbicides to non-targeted organisms and their risk of wider environmental contamination [13]. Herbicide encapsulation is aimed at controlling the liberation of the formulations active ingredient, to enable their use for specific purpose at an intended concentration, reduce the negative impacts they usually have on the environment and maintain the highest possible effectiveness [5,6]. Nanoformulations of herbicide with polymer coating enable its controlled and slower release due to potential interactions between the herbicide and the polymer [12]. A release study of dichlorprop phenoxyherbicides from the interlayer of a $\mathrm{Zn}$ /Al-layered double hydroxide-dichlorprop nanocomposite was also performed by [14] with various concentrations of sodium chloride, sodium carbonate and sodium phosphate and their mixtures in aqueous solution to study their release behavior. The 
application of nanoherbicides is linked with a reduced environmental impact with simultaneous preservation of herbicidal effectiveness [12].

Table 1. Some carrier systems used in formulating nanoherbicides

$\begin{array}{llll}\text { Entry } & \text { Carrier system } & \text { Title }\end{array}$

$1 \quad$ Chitosan and tripolyphosphate

2

Chitosan and Starch

4

Alginate

Chitosan

5
Chitosan/tripolyphosphate nanoparticles loaded with paraquat herbicide: An environmentally safer alternative for weed control The influence of the aquatic humic substances on the colloidal stability and toxicity.

Alginate Reinforced Chitosan and Starch Beads in Slow Release Formulation of Imazaquin HerbicidePreparation and Characterization.

Development and Evaluation of a Controlled Release System of TBH Herbicide Using Alginate Microparticles

Nanoparticles based on Chitosan as Carriers for the Combined Herbicides Imazapic and Imazapyr
Chitosan nanoparticles loaded the herbicide paraquat: Chitosan

Combined Herbicides Imazapic and Imazapyr

\section{Nanotechnology}

Nanotechnology involves the use and manipulation of matter at a tiny scale. At this size, atoms and molecules work differently, and provide various surprising and interesting uses. The emergence of nanotechnology has provided an extensive research in recent years by intersecting with various other branches of science and forming impact on all forms of life [1]. This concept was first begun with lecture delivered by Richard Feynman in 1959. It is a field of science, which deals with production, manipulation and use of materials ranging in nanometers [17]. In nanotechnology, nanoparticles research is an important aspect due to its innumerable applications. In general, NPs are described as particles having diameter sizes less than or equal to $0.1 \mu \mathrm{m}(100 \mathrm{~nm})$ and with specific properties that depend mainly on their size [18]. A study carried out by Krichevskii showed that the nanoparticles have different mode of entry and transport pathways in the environment [19].

Nanomaterials are very important as their properties differ significantly from those of their bulk materials [1]. Entirely novel and enhanced characteristics such as size, distribution and morphology have been revealed by these particles in comparison to the larger particles of the mass material that they have been prepared from [20]. The exotic properties of nanomaterials are largely due to the quantum confinement of electrons within a nanometer sized potential well. These materials are also made in a unique way, stacked atom by atom or atomic layer by layer [21]. Nanotechnology deals with the production and stabilization of various types of nanoparticle [22]. It provides an excellent platform and as vehicle for delivery of active materials to the targeted site without effecting the activity of active material [23]. Nanotechnology as a new branch in science provides new agrochemical technology and new pesticide delivery mechanisms in order to improve crop production, and possibly reduce intensive pesticide usage. It opens up novel applications in agriculture [1]. Nano-formulations are generally expected to increase the apparent solubility of poorly soluble active ingredients, to release the active ingredient in a slow or targeted manner, and to protect against premature degradation [24]. Nanostructure mediated herbicide delivery, enhances its bioavailability, improves the timed release of the active ingredient, and enables precised targeting [8,25]. 


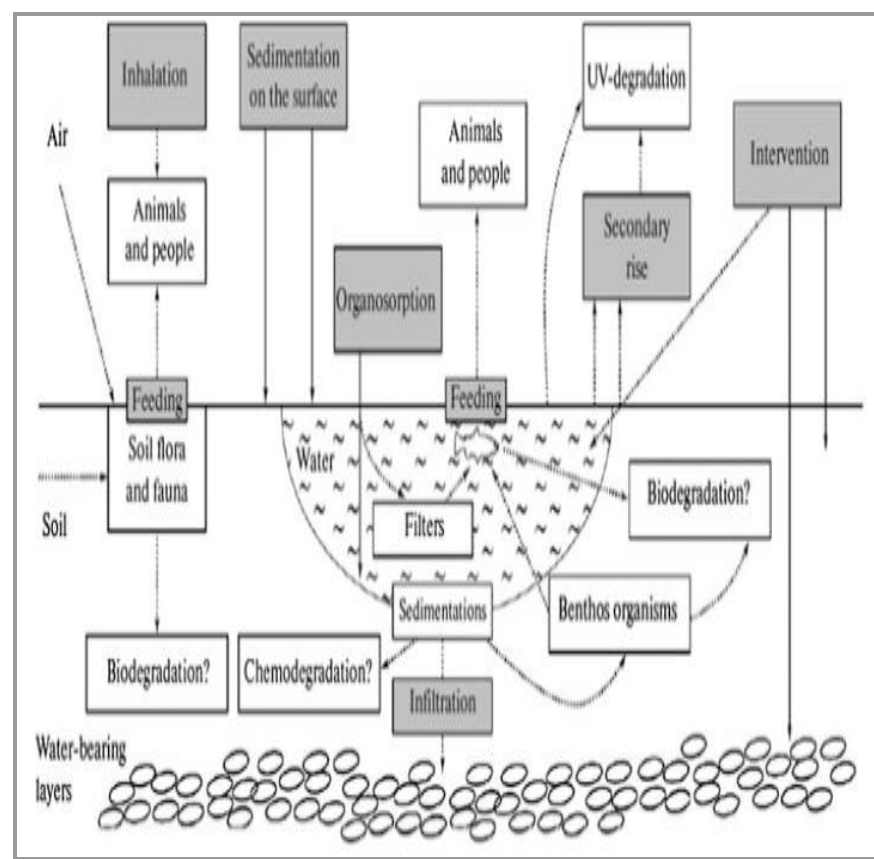

Figure 1. Possible mode of entry and transport pathways of nanoparticles experimentally proven (solid line) and supposed pathways (dotted line) [19].

\subsection{Nonotechnology in Agriculture}

The application of nanotechnology to agriculture and food industries is recently gaining attention. Investments in agriculture and food nanotechnologies carry increasing weight because of their potential benefits ranging from improved food quality and safety to reduced agricultural inputs and improved processing and nutrition [1]. Changes in agricultural technology have been a major factor shaping modern agriculture. Among the latest line of technological innovations, nanotechnology occupies a prominent position in transforming agriculture and food production [26]. It has the capacity to protect plants and monitor its growth, detect animal and plant diseases, enhance food quality, increase global food production, and reduces waste in order to enhance agricultural sustainability [26,27].

Nanotechnology has the potential to revolutionise the agricultural and food industry with novel tools for the molecular management of diseases, rapid disease detection, enhancing the ability of plants to absorb nutrients, among others. The development of nanodevices and materials open up novel applications in plant biotechnology and agriculture. the more efficient and targeted use of inputs, new toxin formulations for weed and pest control, new crop and animal traits, and the diversification and differentiation of farming practices and products within the context of large scale and highly uniform systems of production [28]. Nanotechnology provides new agrochemical agents and new delivery mechanisms to improve crop productivity, and it promises to reduce pesticide use [1].

The key focus areas for nanotechnology in agricultural research are:

- Nanogenetic manipulation of agricultural crops

- Agricultural diagnostics, and drug delivery

- Controlled release of nanofertilizers and nanocomplexes

- Nano-Biosensors

- Nanopesticides and nanoherbicides

- Nano-Bio farming

Table 2. Advantages of using Nanotechnology in the Formulation of Conventional Herbicides

Entry Processes Advantages $\quad$ Ref.

$1 \quad$ Controlled release formulations

Nano-based formulation may allow herbicides to intelligently control the release speed of its active ingredient.

$2 \quad$ herbicide uptake efficiency

3

Modes of controlled release

4

Duration of a.i release
Rate of herbicide loss to the environment
Nano-based formulation may increase herbicide efficiency and also save herbicide resource.

Both the release rate and release pattern of herbicides can be controlled through its encapsulation using a semi-permeable membrane coating.

Nano-based formulation of herbicides can extend effective duration of the herbicide active ingredient in soil.

Nano-based formulation of herbicides can reduce its rate of loss into soil by runoff or leaching. 


\subsection{Nanocapsules for efficient delivery of agrochemicals}

Nanoencapsulation or encapsulation of active ingredient with nanoparticles is a means by which chemicals like pesticides are slowly but efficiently released to a particular host plant for pest control using nanoparticles [10]. It allows for proper absorption of the chemicals into the plants and is currently the most promising technology for the protection of host plants against all agricultural pests [28]. It helps slow release of a chemical to the particular host for weed and insect pest control. The release mechanisms involved in nanoencapsulation of herbicides include diffusion, dissolution, biodegradation and osmotic pressure with specific $\mathrm{pH}[10,25,26]$. With this technology, it is possible to step down the release of the herbicide active ingredient under controlled conditions, thereby reducing currency in application dosage and improving efficiency [29]. Recent study shows that, most leading chemical companies focus on formulation of nanoscale pesticides for delivery into the target host tissue through nanoencapsulation [28]. Herbicides encapsulation in polymeric core shells of nanoparticles can lead to a safer and more convenient management of herbicides that enhances safety of the environment [12]. In a research by Maruyama et al. [13], the study proves that encapsulation of herbicides improved their mode of action and also reduced their toxicity, which indicates their suitability for use in future practical applications. The release kinetics experiments from the study show that the encapsulated herbicides were released more slowly than their free forms. Technologies such as encapsulation and controlled release methods have revolutionized the use of pesticides and herbicides. Pesticides inside nanoparticles are being developed that can be timely released or have release linked to an environmental trigger. Combined with a smart delivery system, herbicide could be applied only when necessary, resulting in greater production of crops and less injury to agricultural workers. Many companies make formulations, which contain nanoparticles within the size ranges of $100-250 \mathrm{~nm}$; they are able to dissolve in water more effectively than existing ones thus increasing their activity [28].

\section{Controlled release formulations}

Controlled release formulations (CRFs) represent an alternative to the conventional systems of herbicide application where the active agent is available for a specific product in duration designed to achieve the intended effect [5]. In a recent term, CRFs or delivery system is a combination of biologically active agent and excipient, usually a polymer is planned to allow delivery of the agent to the target at controlled rates over a specified period $[12,30]$. The herbicide CRFs may be defined as a technology wherein an active ingredient is available for a specific goal at a concentration and with a duration designed to achieve the intended effect, aiming to reach optimal biological effectiveness and to reduce any harmful effects [31]. Controlled release as it relates to agrochemicals is fast becoming the centre of intensive research in agricultural practices [12]. It is now a generally accepted fact that this technology can contribute positively to human's effort in fighting against pest, weed, disease and hunger $[12,30]$.

In conventional application methods, pesticides are always prone to leaching, evaporation, and degradation, which may be photolytic, hydrolytic, or microbial. All these pesticide's environmental fate may remove pesticide active ingredient from their target before they can carry out their purpose [3]. The purpose of using controlled release technology in agriculture is to decrease the excessive impact of the conventional method and to prolong the protective use of agrochemicals or biocides by maintaining an effective concentration over a given interval of time as this will reduce herbicide residues and reduces costs for farmers as well as for companies [8]. A photoresponsive controlled pesticide release film system was constructed by a report in 2019 [32] using PEG supported spiropyran as a carrier and chitosan as a filmforming additive. The pesticide release was observed from the photoresponsive controlled pesticide release film. Their conclusion shows that the work would promote the development of controlled-release pesticide systems. In another study by Bai et al. [4], a novel pesticide carrier LA-NSM (lauric acid-modified Nitraria seed meal) with controlled release property was prepared through grafting esterification of lauric acid onto Nitraria seed meal substrates [4].

Until now, studies carried out on controlled release of herbicides and other pesticides show that this system only allows for a very simple, effective, safer and slower release of pesticide active ingredient in preference to a quick release based on environmental requirements $[33,30]$.

\subsection{Nanocapsules for efficient delivery of agrochemicals}

- They may reduce herbicide loss due to lixiviation, volatilisation, drift and degradation soils.

- They may reduce phytotoxicity.

- They may facilitate herbicide management and safer applications, reducing toxicity in humans.

- They offer the possibility to act more selectively on the intended target. 
- They increase the duration of activity and the effectiveness of the active ingredient, in soil as well as at the foliar level [5].

\section{Chitosan as a matrix for controlled release formulations}

Chitosan (Cts) polymers are naturally occurring polymers, which are obtained by deacetylazation of chitin; and are derived from complex amino polysaccharides by semi-synthetic means. They are cationic polysaccharides composed of $\beta-(1-4)$-linked D-glucosamine and $N$-acetyl-D-glucosamine units. Chitosan is considered as a nontoxic, biocompatible, bioactive, biodegradable polymer, environmentalfriendly material with many superior properties and momentous amounts of amine and hydroxyl groups that can be easily engineered [34]. When the degree of deacetylation of chitin reaches about 50\% (depending on the origin of the polymer), it becomes soluble in aqueous acidic media and is called chitosan [35]. Polysaccharides $50 \%$ deacetylation seems to be most effective for adsorption since functional amine groups are available for interaction. According to [36], chitosan is widely used as encapsulating agent and is widely reported for preparing micro and nanoparticles, because of its cationic charge, biocompatibility and low toxicity, chitosan has been used as a vehicle system for delivery of genes, proteins (including antibodies) and various categories of drugs [25]. Chitosan has also become a valuable vehicle for controlled delivery of agrochemicals and genetic materials because of its proven biocompatibility, biodegradability, nontoxicity, and adsorption abilities [17,37].

Chitosan has free amino groups in its structure, and it can act as better chelating agent than chitin [39].
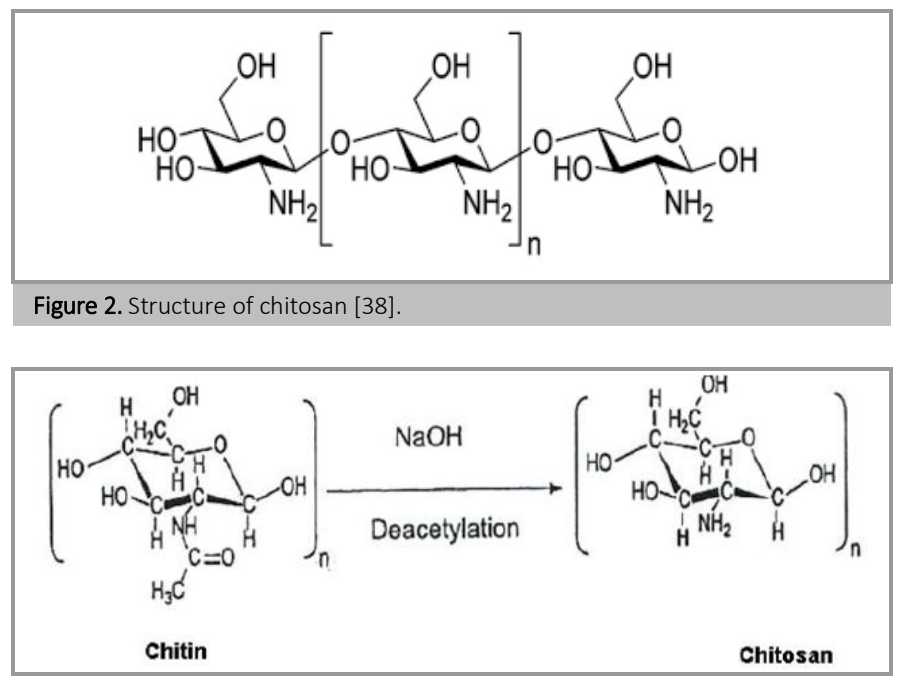

Figure 3. Deacetylation of chitin to chitosan

To reduce the environmental impact from the use of herbicides, a research was carried out by our team [10] using chitosan for the synthesis of pendimethalin copper-chitosan nanoparticles as a controlled release formulation. Chitosan was used as stabilizer and capping agent to develop a controlled release herbicide. In another research by Nnamonu et al. [40], chitosan and starch were reinforced with alginate and employed in compounding slow release formulations of imazaquin herbicide [41]. Developed composite gel composed of carboxymethyl-chitosan (CM-chit) and bentonite (H-bent) used as carrier for encapsulating atrazine and imidacloprid to control their release in water and retard their leaching in soil. According to this research, composite carrier could reduce the potential leaching of pesticides, which can be of benefit in diminishing the negative impact of pesticides in the environment. Results from the work of Dos Santos Silva and co-workers [42], show that the involvement of paraquat with alginate/chitosan nanoparticles alters the release profile of the herbicide, as well as its interaction with the soil, indicating that this system could be an effective means of reducing negative impacts caused by paraquat. The formulation of paraquat with alginate/chitosan nanoparticles is therefore a promising potential for future use in agricultural applications, as it offers increased duration of action of the herbicide's active ingredient on specific targets, while reducing environmental toxicity problems [42].

In agriculture, chitosan finds application in plant defensive mechanism, stimulates plant growth, seed coating, frost protection, and time (controlled/slow) release of pesticides, fertilizers and nutrients into the soil [35]. Major advantages of the encapsulating agrochemicals and genetic material in a chitosan matrix include its ability to function as a protective reservoir for the active ingredients, protecting the ingredients from the surrounding environment while they are in the chitosan domain, controlling their release which allows them to serve as efficient gene delivery systems for plant transformation or controlled release of pesticides [37].

\section{Conclusion}

The intensive use of herbicides and other plant protection products (for agricultural activities) has increased significantly. This increment is to be able to provide adequate food for the over 7 billion and still growing human population. In an attempt to reduce the environmental impact associated with the use of herbicides, the use of controlled release formulations in nanometric size can be a possible alternative to conventional application methods. This is because, the herbicide active ingredient will be available for specific delivery to the target source at a lesser concentration and the duration designed to meet the intended effect, with the aim of reaching optimal biological effectiveness and thereby reducing any harmful effects 
on the environment. Bearing in mind, the aim of this recent approach is majorly to minimize farmers' demand for pesticides to gradually achieve more effective and safer pesticide usage through intelligent design that slows and controls the release of pesticides.

\section{References}

[1] Sekhon, B. S. (2014). Nanotechnology in agri-food production: an overview. Nanotechnology, science and applications, 7, 31-35.

[2] PRD (2017). 2017 World Population Data Sheet with a Special Focus on Youth; Population Reference Bureau.

[3] Rana, S.S. and Rana, M.C. (2015). Advances in Weed Management. Department of Agronomy, College of Agriculture, CSK Himachal Pradesh Krishi Vishvavidyalaya, Palampur, Pp. 55.

[4] Bai, B., Xu, X., Hai, J., Hu, N., Wang, H., \& Suo, Y. (2019). Lauric Acid-Modified Nitraria Seed Meal Composite as Green Carrier Material for Pesticide Controlled Release. Journal of Chemistry, 2019.

[5] Sopeña, F., Maqueda, C., \& Morillo, E. (2009). Controlled release formulations of herbicides based on microencapsulation. Ciencia e investigación agraria, 36(1), 27-42.

[6] Hashim, N., Muda, Z., Hamid, S. A., Isa, I. M., Kamari, A., Mohamed, A., ... \& Ghani, S. A. (2014). Characterization and controlled release formulation of agrochemical herbicides based on zinclayered hydroxide-3-(4-methoxyphenyl) propionate nanocomposite. $J$ Phys Chem Sci, 1, 1-6.

[7] Aouada, F. A., de Moura, M. R., Orts, W. J., \& Mattoso, L. H. (2010). Polyacrylamide and methylcellulose hydrogel as delivery vehicle for the controlled release of paraquat pesticide. Journal of Materials Science, 45(18), 4977-4985.

[8] Onyido, I., Sha'Ato, R., \& Nnamonu, L. A. (2012). Environmentally friendly formulations of trifluralin based on alginate modified starch. Journal of Environmental protection, 3(09), 1085.

[9] Mahmood, K., Suleman, M., Hassan, S., \& Badshah, S. K. (2013). Assessment the leaching potential of pendimethalin and its transformation products in soil. World Applied Sciences Journal, 26(5), 583-587.

[10] Itodo, H. U., Nnamonu, L. A., \& Wuana, R. A. (2017). Green Synthesis of Copper Chitosan Nanoparticles for Controlled
Release of Pendimethalin. Asian Journal of Chemical Sciences, 1-10.

[11] Koterba, M. T., Banks, W. S. L., \& Shedlock, R. J. (1993). Pesticides in shallow groundwater in the Delmarva Peninsula. Journal of Environmental Quality, 22(3), 500-518.

[12] Jampílek, J., \& Král'ová, K. (2015). Applications of nanoformulations in agricultural production and their impact on food and human health. Proceedings of ECOpole, 9.

[13] Maruyama, C. R., Guilger, M., Pascoli, M., Bileshy-José, N., Abhilash, P. C., Fraceto, L. F., \& De Lima, R. (2016). Nanoparticles based on chitosan as carriers for the combined herbicides imazapic and imazapyr. Scientific reports, 6, 19768.

[14] Hashim, N., Sharif, S. N. M., Muda, Z., Isa, I. M., Bakar, S. A., Ali, N. M., ... \& Mamat, M. (2019). Release Behavior Of Dichlorprop From Zn/Al-Ldh-Dichlorprop Nanocomposite Into Chloride, Carbonate And Phosphate Solutions. Jurnal Teknologi, 81(2).

[15] Grillo, R., Pereira, A. E., Nishisaka, C. S., de Lima, R., Oehlke, K., Greiner, R., \& Fraceto, L. F. (2014). Chitosan/tripolyphosphate nanoparticles loaded with paraquat herbicide: an environmentally safer alternative for weed control. Journal of hazardous materials, 278, 163-171.

[16] Grillo, R., Clemente, Z., de Oliveira, J. L., Campos, E. V. R., Chalupe, V. C., Jonsson, C. M., ... \& Oehlke, K. (2015). Chitosan nanoparticles loaded the herbicide paraquat: the influence of the aquatic humic substances on the colloidal stability and toxicity. Journal of hazardous materials, 286, 562-572.

[17] Kavitha, K. S., Baker, S., Rakshith, D., Kavitha, H. U., Yashwantha Rao, H. C., Harini, B. P., \& Satish, S. (2013). Plants as green source towards synthesis of nanoparticles. Int Res J Biol Sci, 2(6), 66-76.

[18] Hanemann, T., \& Szabó, D. V. (2010). Polymer-nanoparticle composites: from synthesis to modern applications. Materials, 3(6), 3468-3517.

[19] Krichevskii, G. E. (2010). Nanotechnologies: dangers and risks. Inspecting principles for nano technologies and nanomaterials. Nanotekhnol Okhrana Zdorov'ya, 2(3), 4. 
[20] Dai, J., \& Bruening, M. L. (2002). Catalytic nanoparticles formed by reduction of metal ions in multilayered polyelectrolyte films. Nano Letters, 2(5), 497-501.

[21] Adak, T., Kumar, J., Shakil, N. A., \& Walia, S. (2012). Development of controlled release formulations of imidacloprid employing novel nano-ranged amphiphilic polymers. Journal of Environmental Science and Health, Part B, 47(3), 217-225.

[22] Vidyasagar, G. M. (2014). Green synthesis, characterization and antimicrobial activity of Silver Nanoparticles by using Sterculia foetida L. young leaves aqueous extract.

[23] Nikhil N. S., Samrat K. S. and Karthick R. N. (2014). Preparation of Copper Nanoparticles Incorporated Fluconazole for the Improved Antifungal Activity against Human Pathogenic Fungi. International Review of Applied Biotechnology and Biochemistry: 2 (1): 251-259.

[24] Kah, M., Beulke, S., Tiede, K., \& Hofmann, T. (2013). Nanopesticides: state of knowledge, environmental fate, and exposure modeling. Critical Reviews in Environmental Science and Technology.

[25] preet Kaur, S., Rao, R., Hussain, A., \& Khatkar, S. (2011). Preparation and characterization of rivastigmine loaded chitosan nanoparticles. Journal of Pharmaceutical sciences and Research, 3(5), 1227.

[26] Biswal, S. K., Nayak, A. K., Parida, U. K. and Nayak P. L. (2012). Applications of Nanotechnology in Agriculture and Food Sciences. International Journal of Science, Innovation and Discovery. 2 (1): 21-36.

[27] Prasad, R., Kumar, V., \& Prasad, K. S. (2014). Nanotechnology in sustainable agriculture: present concerns and future aspects. African Journal of Biotechnology, 13(6), 705-713.

[28] Prasad, R., Kumar, V., \& Prasad, K. S. (2014). Nanotechnology in sustainable agriculture: present concerns and future aspects. African Journal of Biotechnology, 13(6), 705-713.

[29] Scrinis, G., \& Lyons, K. (2007). The emerging nano-corporate paradigm: nanotechnology and the transformation of nature, food and agri-food systems. International Journal of Sociology of Food and Agriculture, 15(2), 22-44.

[30] Huang, B., Chen, F., Shen, Y., Qian, K., Wang, Y., Sun, C., ... \& Cui, H. (2018). Advances in targeted pesticides with environmentally responsive controlled release by nanotechnology. Nanomaterials, 8(2), 102.

[31] Rüegg, W. T., Quadranti, M., \& Zoschke, A. (2007). Herbicide research and development: challenges and opportunities. Weed Research, 47(4), 271275.

[32] Qing X., Guohua L., Yuanjing Z., and Fei N. (2019). Preparation and Properties of Photoresponsive Controlled Release Pesticide Film. Advances in Engineering Research, 181:230-233.

[33] Faria, D. M., Júnior, D., Macias, S., Nascimento, J. P. L. D., Nunes, E. D. S., Marques, R. P., ... \& Moreto, J. A. (2017). Development and evaluation of a controlled release system of TBH herbicide using alginate microparticles. Materials Research, 20(1), 225-235.

[34] Usman, M., Ibrahim, N., Shameli, K., Zainuddin, N., \& Yunus, W. (2012). Copper nanoparticles mediated by chitosan: synthesis and characterization via chemical methods. Molecules, 17(12), 14928-14936.

[35] Rinaudo, M. (2006). Chitin and chitosan: properties and applications. Progress in polymer science, 31(7), 603-632.

[36] Chen, C., Gao, Z., Qiu, X., \& Hu, S. (2013). Enhancement of the controlled-release properties of chitosan membranes by crosslinking with suberoyl chloride. Molecules, 18(6), 7239-7252.

[37] Kashyap, P. L., Xiang, X., \& Heiden, P. (2015). Chitosan nanoparticle based delivery systems for sustainable agriculture. International journal of biological macromolecules, 77, 36-51.

[38] Malathi, S., Balakumaran, M. D., Kalaichelvan, P. T., \& Balasubramanian, S. (2013). Green synthesis of gold nanoparticles for controlled delivery. $A d v$ Mater Lett, 4(12), 933-940.

[39] Lakshmi, K. B., \& Sudha, P. N. (2012). Adsorption of Copper (II) ion onto chitosan/sisal/banana fiber hybrid composite. International journal of environmental sciences, 3(1), 453-470.

[40] Nnamonu, L. A., Sha'Ato, R., \& Onyido, I. (2012). Alginate reinforced chitosan and starch beads in slow release formulation of imazaquin herbicide-preparation and characterization. Materials Sciences and Applications, 3(08), 566.

[41] Li, J., Yao, J., Li, Y., \& Shao, Y. (2012). Controlled release and retarded leaching of 
pesticides by encapsulating in carboxymethyl chitosan/bentonite composite gel. Journal of Environmental Science and Health, Part B, 47(8), 795-803.

[42] dos Santos Silva, M., Cocenza, D. S., Grillo, R., de Melo, N. F. S., Tonello, P. S., de
Oliveira, L. C., ... \& Fraceto, L. F. (2011). Paraquat-loaded alginate/chitosan nanoparticles: preparation, characterization and soil sorption studies. Journal of hazardous materials, 190(1-3), 366-374.

How to cite this manuscript: H. U. Itodo*. Controlled Release of herbicides using Nanoformulation: A Review, Journal of Chemical Reviews (J. Chem. Rev.), 2019, 1(2), 130-138. 